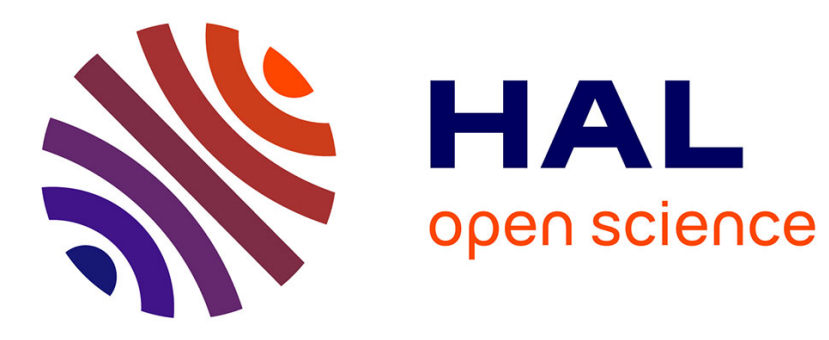

\title{
Le Kirghizstan : soft power et inflation électorale Boris Pétric
}

\section{To cite this version:}

Boris Pétric. Le Kirghizstan : soft power et inflation électorale. Hérodote - Revue de géographie et de géopolitique, 2008, Stratégies américaines aux marches de la Russie, 129 (2), pp.145-165. 10.3917/her.129.0145. halshs-01849814

\section{HAL Id: halshs-01849814 https://shs.hal.science/halshs-01849814}

Submitted on 26 Jul 2018

HAL is a multi-disciplinary open access archive for the deposit and dissemination of scientific research documents, whether they are published or not. The documents may come from teaching and research institutions in France or abroad, or from public or private research centers.
L'archive ouverte pluridisciplinaire HAL, est destinée au dépôt et à la diffusion de documents scientifiques de niveau recherche, publiés ou non, émanant des établissements d'enseignement et de recherche français ou étrangers, des laboratoires publics ou privés. 


\title{
LE KIRGHIZSTAN : SOFT POWER ET INFLATION ÉLECTORALE Boris Petric
}

\author{
La Découverte | « Hérodote »
}

2008/2 n 129 | pages 145 à 165

ISSN 0338-487X

ISBN 9782707154781

Article disponible en ligne à l'adresse :

https://www.cairn.info/revue-herodote-2008-2-page-145.htm

\section{Pour citer cet article :}

Boris Petric, «Le Kirghizstan : soft power et inflation électorale », Hérodote 2008/2 (n¹29), p. 145-165.

DOI 10.3917/her.129.0145

Distribution électronique Cairn.info pour La Découverte.

(C) La Découverte. Tous droits réservés pour tous pays.

La reproduction ou représentation de cet article, notamment par photocopie, n'est autorisée que dans les limites des conditions générales d'utilisation du site ou, le cas échéant, des conditions générales de la licence souscrite par votre établissement. Toute autre reproduction ou représentation, en tout ou partie, sous quelque forme et de quelque manière que ce soit, est interdite sauf accord préalable et écrit de l'éditeur, en dehors des cas prévus par la législation en vigueur en France. Il est précisé que son stockage dans une base de données est également interdit. 


\section{Le Kirghizstan : soft power et inflation électorale}

Boris Petric*

Les événements du mois de mars 2005 au Kirghizstan, baptisés «Révolution des tulipes », ont subitement médiatisé mondialement cette ancienne République soviétique située aux confins de l'ex-URSS et de la Chine. Au cours d'élections législatives, un mouvement populaire de contestation conduit Askar Akaev à quitter le pouvoir et finalement le pays ${ }^{1}$. Les grands médias internationaux y ont vu un mouvement populaire spontané et massif réclamant l'instauration de la démocratie face à un régime de plus en plus autoritaire. La société civile se serait soulevée brutalement contre la tyrannie de son gouvernement pour demander davantage de droits politiques. Cette interprétation repose sur une vision «naturalisante» des phénomènes sociaux. Elle occulte les motivations socio-économiques de la mobilisation populaire et minimise les nouvelles influences qui ont permis à de nouveaux acteurs d'émerger sur l'échiquier politique kirghize depuis son émancipation. Un tel scénario intervient suite à l'ouverture massive du pays sur l'extérieur et à la réalisation de profonds changements effectués depuis 1991. Au niveau politique, cette «révolution » consacre les réformes inspirées par l'aide internationale au développement et certaines chancelleries occidentales très investies dans l'assistance à la démocratie. Ces acteurs ont tout d'abord contribué à un déplacement du pouvoir de l'administration présidentielle vers le Parlement. Son nouveau rôle s'incarne précisément par l'importance prise par la compétition électorale dans la vie politique kirghize. Depuis 1998, toute une série d'élections ont permis progressivement la mise en place d'un dispositif élaboré, par l'intermédiaire d'un long travail « sociétal », par de nombreux acteurs extérieurs. Cependant

* Anthropologue, chargé de recherche au CNRS (IIAC/LAIOS).

1. Askar Akaev réside actuellement à Moscou. 
l'instauration de la démocratie ne se réduit pas à la multiplication des compétitions électorales.

Après le retrait de l'ancien centre moscovite, le Kirghizstan entame un processus radical de transformations politiques qui lui vaut rapidement d'être considéré comme le bon élève de la région auprès des organisations internationales. Une multitude d'organisations internationales, de fondations et d'ONG y déferlent pour prendre en charge la libéralisation économique et politique du pays, voyant même dans le Kirghizstan un laboratoire expérimental pour instaurer la nouvelle idéologie en vigueur depuis l'effondrement du mur de Berlin : la bonne gouvernance. En matière économique, le Kirghizstan opte officiellement pour une thérapie de choc (Abazov, 1999), dont la politique d'ajustements structurels (Stiglitz, 2001) prônés par la Banque mondiale et le FMI. Cela implique une privatisation élargie et le désengagement massif de l'État de certains secteurs d'activité. De plus, l'aide internationale consacre un effort particulier pour développer le secteur dit de la «société civile » qui se matérialise par une prolifération d'ONG locales, qui seraient selon le PNUD plus de $11000^{2}$ en 2006. Comme le souligne un de ces représentants, Edil Baisalov ${ }^{3}:$ «Si on dit que la Hollande est le pays des tulipes, le Kirghizstan est le pays des ONG. » Ce trentenaire, héros de la Révolution des tulipes, est à la tête d'une coalition d'ONG, Koalitsia ${ }^{4}$. Cette organisation a joué un rôle déterminant lors de la Révolution des tulipes en organisant une observation électorale. Comment peut-on interpréter l'émergence d'ONG comme Koalitsia ${ }^{5}$ et la prolifération d'ONG locales (plus de 11000 ) au Kirghizstan? S'agit-il de l'expression du « réveil » d'une société civile kirghize atomisée à l'époque soviétique ou assiste-t-on à l'instauration d'une nouvelle forme de gouvernementalité qui illustre un phénomène plus vaste à l'échelle mondiale : la transnationalisation des espaces politiques?

\section{De la tutelle moscovite à l'encadrement international}

Après avoir été une zone particulièrement fermée à l'époque soviétique, le Kirghizstan s'est massivement ouvert vers l'extérieur. Cette ancienne République soviétique, qui était l'une des plus pauvres de l'Union, a choisi de lutter contre son enclavement en favorisant les flux des hommes, des idées et des biens. En quelques

2. www.undp.kg.

3. Entretien personnel réalisé en juillet 2005.

4. http ://www.civilsoc.org.

5. Ce travail repose essentiellement sur des matériaux et des sources empiriques qui ont été collectés par l'auteur au Kirghizstan au cours de plusieurs séjours de terrain depuis 2000. 
années, elle devient le théâtre d'un nouveau grand jeu sur lequel se déploient différentes techniques d'influences des grandes puissances, dont le soft power américain qui repose notamment sur l'action d'ONG et de fondations. Cette action est relayée par un certain nombre d'organisations internationales (PNUD, OSCE, etc.) qui partagent une vision commune sur les réformes à mener dans ce pays.

Il est important de rappeler que la tutelle internationale et les programmes d'assistance s'amorcent avant tout parce que Moscou décide d'abandonner l'Asie centrale. À la fin des années 1980, un consensus se dégage parmi les élites à Moscou : l'Asie centrale est un boulet dont il faut se délester. Les accords de Bieloviesk ${ }^{6}$ signés entre l'Ukraine, la Biélorussie et la Fédération de Russie viennent consacrer cette vision. De nombreux députés de la Douma proches de Boris Eltsine pensent que le système soviétique a périclité à cause des Républiques du Sud (Caucase et Asie centrale), responsables d'une généralisation de la corruption dans l'ensemble de l'Union, comme le prouve le gigantesque scandale du coton en Ouzbékistan qui éclabousse l'ensemble du système. La Russie de Boris Eltsine se désengage progressivement de l'Asie centrale tout en maintenant une présence grâce à des accords politiques et militaires, sans pour autant garder une influence sur le tissu social. L'étranger proche n'est pas la priorité pour le gouvernement et la culture d'influence russe consiste à penser que l'on agit légitimement en entretenant uniquement des rapports avec les élites. De plus, les nombreux Russes de ces républiques ne font pas l'objet d'une attention particulière de la part de Moscou. Ces «pieds rouges » quittent massivement ces pays, contribuant à accentuer l'érosion de la présence de l'ancien centre. Les citoyens de nationalité russe qui choisissent de rester en Asie centrale ne sont pas organisés politiquement et ne jouent pas un rôle dans la vie politique de ces pays. Moscou ferme les portes de ses plus grandes universités aux jeunes élites centrasiatiques, s'amputant ainsi d'un des éléments clés de l'influence dans le monde d'aujourd'hui. Les élites kirghizes, comme celles de toutes les républiques centrasiatiques, doivent alors gérer une indépendance qu'elles n'ont pas réclamée ${ }^{7}$.

Dans ce contexte, ce petit pays qui compte 5 millions d'habitants pour une superficie de $200000 \mathrm{~km}^{2}$ (soit 1/3 de la France) fait l'objet d'une attention internationale importante. Enclavé entre la Chine, le Kazakhstan et l'Ouzbékistan, il ne recèle pas de ressources gazières ou pétrolières, mais sa proximité avec la Chine, l'Afghanistan et la Russie lui confère une position géo-stratégique cruciale. Sa géographie est caractérisée par un fort contraste entre le nord du pays,

6. Ces accords matérialisent la mort de l'URSS.

7. Contrairement à la situation dans certains pays de l'Est, il n'y a pas eu de mouvements populaires pour réclamer l'indépendance. 
montagneux, riche en ressources hydriques et pauvre en terres arables, et une partie méridionale, la vallée de Ferghana, constituée d'une vaste plaine agricole. En zone rurale, la population du Nord est tournée vers les activités d'élevage même si celles-ci ont considérablement pâti de la privatisation. La densité de la population y est très faible tandis qu'au Sud une forte population plus anciennement sédentarisée se concentre dans une zone où les conflits pour le partage des terres arables et de l'eau sont grandissants. Le contraste géographique se retrouve également dans les représentations identitaires. Les Méridionaux (juzhanin) se différencient des Nordistes (severanin) notamment en soulignant un rapport différent à l'islam, qui serait plus distant au Nord. Par ailleurs, il existe aussi un contraste ethnique à l'intérieur de la vallée de Ferghana, où se concentre une forte minorité ouzbèke. Pour de nombreux analystes, la vallée de Ferghana constitue un des foyers ethnico-religieux les plus explosifs du monde et un haut lieu d'expansion des mouvements transnationaux islamistes. Cette réputation semble disproportionnée, mais la communauté internationale suit de près l'évolution de deux mouvements islamistes dans la région: le Hizb-ut-Tahrir, qui souhaite supprimer les frontières soviétiques qu'il juge artificielles afin de créer un grand califat d'Asie centrale réunissant tous les musulmans dans une même entité politique; et une organisation militaire, le MOI - le Mouvement islamique d'Ouzbékistan - de Djuma Namangani, qui œuvre en Afghanistan auprès des Talibans et d'Al-Qaida tout en recrutant au Kirghizstan et dans la région.

Pour les organisations internationales, le Kirghizstan apparaît comme un nouveau terrain de prédilection afin d'élargir son champ d'action en matière d'aide au développement. La République kirghize est immédiatement considérée comme un laboratoire en matière de réformes à instaurer dans les pays dits en transition. Les organisations internationales se fixent comme objectif d'éradiquer le modèle soviétique et de promouvoir la nouvelle norme économico-politique en vigueur : la bonne gouvernance. Les pays en transition doivent adopter la démocratie et l'économie de marché. Avant d'être tombé en disgrâce auprès des bailleurs de fonds quelques mois avant la Révolution des tulipes (mars 2005), le pays bénéficie de très nombreux programmes destinés à favoriser le processus de démocratisation. La République kirghize est alors présentée comme un îlot démocratique dans un environnement régional autoritaire, lui valant le surnom de Suisse de l'Asie centrale.

Du côté kirghize, l'adoption du nouveau catéchisme international part d'une considération pragmatique. Le président Askar Akaev ${ }^{8}$ comprend que son petit

8. Il a théorisé sous le vocable « $i$ » (« et et ») sa conception de la politique extérieure multipolaire qui consiste à trouver un équilibre avec les grandes puissances notamment russes et américaines. 
pays, qui était l'une des Républiques soviétiques les plus pauvres, pâtit de l'effondrement du système soviétique. L'enclavement de la région et la rareté des ressources l'obligent immédiatement à encourager une politique étrangère volontariste à l'égard des institutions internationales et des puissances occidentales. Il se présente comme le champion des réformes et attire ainsi de très nombreuses organisations et ONG internationales. Dans ce contexte, le Kirghizstan devient un terrain idéal pour la diffusion de la technique d'influence américaine du soft power $^{9}$. Pour les Kirghizes, les programmes de développement s'apparentent à une rente qu'il faut capter. Il ne s'agit plus d'attirer les subventions issues de l'Union soviétique ${ }^{10}$ mais celles de l'aide internationale. Dans un pays enclavé où l'économie piétine (Pomfret, 2003), le Kirghizstan devient alors une société de captation de ressources. Cette ouverture officielle (Akayev, 1995) dissimule cependant de fortes résistances et une volonté de la part des élites kirghizes de garder une influence déterminante sur l'évolution du pays.

Dans un premier temps, une approche institutionnelle des réformes se concrétise par des changements législatifs déterminants, car elle modifie le jeu politique en attribuant un rôle prépondérant au Parlement (Jogorku Kenesh). Ces modifications se réalisent dans un contexte de pression internationale à l'encontre de Bichkek, qui accueille alors tout un ensemble de programmes financés par l'Union européenne, les USA, le PNUD, l'OSCE et différentes organisations transnationales. Le nouveau statut du Parlement implique rapidement la tenue d'élections législatives en 1995. Cependant, au cours des premières années de l'indépendance, l'appareil présidentiel continue à garder la mainmise sur les affaires politiques. Ces premières élections « libres » vont permettre l'émergence de nouveaux acteurs politiques. Quelques députés se présentent officiellement comme constituant l'opposition. Un groupe se forme notamment autour d'un député, Azimbek Beknazarov, originaire du sud du pays. Ce dernier joue un rôle important au moment du référendum de 2001 en organisant une série de protestations à Bichkek et dans le sud du pays contre le président Akaev, qui est soupçonné d'avoir passé un accord secret avec la Chine visant à «brader» au grand voisin une bande territoriale frontalière de plusieurs milliers d'hectares (Laruelle, Peyrouse, 2006). Les protestations tournent à l'affrontement dans la circonscription du député (Aksy) et marquent un tournant dans la vie politique kirghize. Le Premier ministre de

9. Le soft power désigne l'action américaine autour de différents acteurs agissant à l'étranger à travers l'activité d'ONG, de fondations privées ou parapubliques finançant l'émergence d'acteurs locaux.

10. La République kirghize était l'une des Républiques les plus subventionnées par le centre à l'époque soviétique. 
l'époque, Kurmanbek Bakiev, lui aussi originaire du Sud, quitte le gouvernement ${ }^{11}$. Quelques années plus tard, c'est autour de ce dernier et de Roza Otumbaeva que va se constituer une coalition de partis politiques d'opposition pour renverser Akaev. Cet événement met en lumière la logique politique kirghize, fortement marquée par une opposition régionale à l'intérieur du pouvoir qui existait déjà à l'époque soviétique. Au-delà d'un embryon d'opposition, c'est avant tout un jeu subtil qui structure les rapports de pouvoir entre factions régionales opposant le sud et le nord du pays.

La réduction du nombre de sièges au nouveau Jogorku Kenesh ( 75 députés) ${ }^{12}$ et la politique de désengagement de l'État accentuent la compétition au sein de la nébuleuse présidentielle pour obtenir des positions de pouvoir. Le mandat législatif devient alors un enjeu considérable dans la société kirghize car il permet de devenir un acteur politique qui s'autonomise à l'égard des contingences politiques du moment en bénéficiant de l'immunité. Cette réforme est réalisée sous l'impulsion du PNUD, de l'OSCE et aussi d'une organisation américaine, IFES ${ }^{13}$, à la pointe des questions de réformes des systèmes électoraux. Elle a pu se concrétiser car les élites en place ont vu dans la montée en puissance du Parlement la possibilité d'asseoir leur influence dans la nouvelle société kirghize en pérennisant la privatisation. Devenir député au sein du nouveau Parlement offre en effet la possibilité de bénéficier d'une immunité parlementaire nécessaire à des élites qui se sont enrichies dans des conditions souvent à la limite de la légalité. À partir de 1995, la tenue des élections devient un événement incontournable qui ne se réduit pas à un affrontement entre une nébuleuse présidentielle et une opposition clairement identifiée. De manière générale les élites, qu'elles soient du Sud ou du Nord, ayant profité de la privatisation des ressources, s'affrontent pour conquérir le Parlement qui est devenu un lieu de pouvoir déterminant, comme en témoigne le scrutin de mars 2005 qui va conduire le président à la démission.

Il faut souligner ici que, dans d'autres pays voisins comme le Turkménistan ou l'Ouzbékistan, les élections ne jouent pas ce rôle de catalyseur car leur Parlement ${ }^{14}$ n'est qu'une institution venant valider la politique présidentielle et n'est pas devenu ce lieu politique où s'affrontent différentes forces sociales. Dans ces régimes,

11. Certains prétendent qu'il est parti pour désapprouver la politique répressive d'Akaev, d'autres disent qu'il a été limogé pour sa gestion calamiteuse de ce conflit.

12. On peut noter que l'on assiste à un processus identique dans de très nombreux pays de la zone.

13. L'IFES, International Foundation for Election Systems, est basé à Washington.

14. En Ouzbékistan, le Parlement se réunit pour une session très courte et entérine les grandes lignes de la politique présidentielle. 
l'initiative législative et la responsabilité politique demeurent essentiellement aux mains de l'administration présidentielle. Toute action collective contestataire, sous forme de manifestations, continue à être proscrite. De plus, présenter des candidats à des élections législatives ou présidentielles s'opposant au pouvoir en place est inconcevable. Si le scrutin électoral apparaît comme le moment clé, éphémère, d'une mobilisation collective, les acteurs stratégiques qui donnent corps et cohérence à la contestation émergent au cours d'un long processus qui commence au lendemain de l'indépendance.

Le modèle de réforme donne effectivement davantage d'initiatives législatives au Jogorku Kenesh, dont les députés et le président (speaker) deviennent des figures politiques prépondérantes dans le paysage politique ${ }^{15}$. A partir des élections de 2005, le gouvernement est responsable devant le Parlement. On retrouve cette architecture de réformes, à quelques exceptions près, dans toutes les sociétés postsocialistes, consistant à accroître la responsabilité du Parlement, dont le gouvernement tire sa légitimité, au détriment des appareils présidentiels ${ }^{16}$. Dans ces circonstances, l'organisation d'élections législatives devient à la fois enjeu majeur et moment de tensions.

Outre la pression exercée sur les gouvernements pour permettre la naissance d'un multipartisme, tout un travail est mené en profondeur au niveau institutionnel. Une attention particulière est apportée aux commissions électorales. Le PNUD, par exemple, finance plusieurs programmes concernant l'organisation technique des élections ${ }^{17}$. Pour celles de 2005, un nouveau système informatique, le Shaïlo system, est mis en place pour rationaliser le décompte des voix. De plus, l'IFES ${ }^{18}$ entreprend un travail de longue haleine sur la diffusion du nouveau code électoral, apporte son soutien pour modifier les pratiques électorales ${ }^{19}$ en proposant

15. Signalons également l'apparition d'une nouvelle figure de pouvoir dans tous les pays en transition, l'ombudsman, qui est un médiateur auquel les citoyens peuvent directement s'adresser en cas de conflit avec l'administration ou le gouvernement. Bakir Ulu, l'ombudsman kirghize, s'est présenté contre K. Bakiev aux présidentielles de juillet 2005 ; l'ancien speaker $\mathrm{O}$. Tekebaev est tête de la liste d'opposition Ata Meken qui va mener campagne contre K. Bakiev aux élections législatives de décembre 2007.

16. Cette orientation constitutionnelle entraîne, dans les situations postrévolutionnaires, à confondre le rôle du président avec celui de Premier ministre, comme on le voit actuellement en Ukraine ou au Kirghizstan.

17. http ://www.undp.kg.

18. International Fund For Elections Systems : cette organisation financée par le département d'État est non seulement présente dans l'espace postsoviétique mais aussi dans des pays comme l'Irak ou l'Afghanistan.

19. C'est notamment cette organisation qui a mis en place les urnes transparentes et le système d'empreintes antifraudes. 
des formations aux centaines de commissions électorales locales pour éviter toutes formes de fraudes. On généralise des techniques électorales (urne transparente, caroussel voting - un système antivote multiple -, etc.). Au moment de voter, on vaporise une encre invisible et indélébile sur le pouce pour éviter tout vote multiple. Ce dispositif technique a créé un certain émoi dans la population et quelques rumeurs ont circulé sur la nocivité du produit.

\section{Société civile et ONG locales : une nouvelle chimère ?}

Parallèlement, un travail plus « sociétal » est également entrepris pour favoriser l'émergence d'une «société civile ${ }^{20}$ (Rist, 2002), consistant essentiellement à soutenir l'émergence d'associations non gouvernementales. Les financements proposés par les différentes agences internationales et les grandes ONG permettent la constitution d'un tissu considérable d'ONG locales. Cette approche de la «bonne gouvernance » (Atlani, 2005) n'est pas spécifique au Kirghizstan et s'inscrit dans un accroissement du rôle joué par les organisations internationales non gouvernementales dans les pays en voie de développement. Les ONG locales au Kirghizstan sont inégalement réparties sur le territoire, elles sont de tailles variables et concernent des secteurs d'activité et des groupes de populations tout aussi divers allant de la défense d'une minorité ethnique, à celles des femmes, de l'écologie, des jeunes, des malades du sida, de l'artisanat, d'un projet de micro-crédit, de la gestion des ressources, de la lutte contre le terrorisme, etc. Elles incarnent souvent beaucoup plus les priorités fixées par les agents internationaux que les préoccupations réelles de la population locale. C'est ici que se pose la question de l'usage abusif de la naissance de la société civile. Pour de nombreux bailleurs de fonds et fervents défenseurs du soft power, la multiplication des ONG symbolise l'émergence de forces sociales «d'en bas » (from the bottom) qui s'opposent à l'État et aux élites (top). Leur nombre serait donc un indicateur de la vitalité du corps social kirghize. L'Agence de développement américaine (UsAid), l'une des plus actives au Kirghizstan, pense pouvoir même mesurer le phénomène et déclare: «Les pays de l'Asie centrale sont logiquement classés dans les derniers dans l'index de l'ONG de développement durable. Le Kazakhstan, l'Ouzbékistan, le Tadjikistan et le Turkmenistan sont respectivement classés $21^{\mathrm{e}}, 25^{\mathrm{e}}, 26^{\mathrm{e}}$ et $28^{\mathrm{e}}$. Le Kirghizstan, où commence à émerger une société civile, est classé $11^{\mathrm{e}}$ sur 28 en 2000 . Cepen-

20. L'usage critique de la notion de société civile renvoie à un débat important sur la transition dans l'espace postsocialiste qu'il n'est pas possible de développer ici et que l'auteur a par ailleurs traité dans un autre article ( $c f$. bibliographie). 
dant, même si le savoir-faire et la capacité organisationnelle des ONG locales s'améliorent, l'héritage d'une très faible activité, l'environnement légal et la capacité financière continuent de freiner leur croissance. »

La société civile peut donc se quantifier et certains observateurs analysent les changements de la société kirghize à travers l'importance des ONG : «Le Kirghizstan a une socité civile relativement vivante et la participation des ONG et des médias indépendants à son essor est importante ${ }^{21}$. $\gg$ Cette tendance se généralise et l'ONG américaine Freedom House s'appuie sur ce genre d'indice pour évaluer la liberté dans le monde à travers certains critères, dont le nombre d'ONG comptabilisé dans un pays.

Le foisonnement d'ONG locales est avant tout un indicateur des relations nouées entre la société kirghize et l'aide internationale, et ne peut en aucun cas se réduire à un «réveil naturel de la société civile». Cette évolution reflète également à quel point le champ du pouvoir est devenu transnational et mondial car la plupart des ONG locales s'inscrivent dans des réseaux transnationaux. Le caractère totalisant du phénomène fait qu'il n'y a pas un village qui n'ait pas 2 ou 3 ONG aujourd'hui. La quasi-totalité des 11000 ONG locales kirghizes enregistrées auprès du ministère de la Justice ont la caractéristique d'être exclusivement financées par l'aide internationale. Les organisations non gouvernementales sont indépendantes de l'État, mais se retrouvent donc dans des relations de pouvoir avec d'autres institutions, dont les bailleurs de fonds internationaux. L'un des grands financeurs de l'opération pour l'ensemble des pays de l'Est, George Soros, résume bien la stratégie internationale, pensant qu' «il faut passer dans ces pays-là d'une idéologie de plan à une idéologie de projet». Chaque groupe, chaque village, doit avoir son projet et son $\mathrm{ONG}$, ce qui fait dire à Steven Sampson que se développe dans ces pays postsocialistes une «vie sociale de projets» (Sampson, 2002).

Après le désengagement de l'État dans de très nombreux secteurs d'activités, ces ONG sont susceptibles de prendre le relais de celui-ci pour organiser la vie sociale. Afin de stimuler cette orientation politique, les grands bailleurs de fonds internationaux déversent l'aide à travers ce nouveau partenaire qu'est l'ONG locale. Leur multiplication présente un double intérêt : éviter l'État, jugé incompétent et corrompu, et constituer un réseau d'agents de distribution. Pour ces organisations internationales, les ONG locales seront l'expression de la naissance d'une société civile.

21. $C f$. «International Crisis group », ICG Report, $\mathrm{n}^{\circ} 84,2004$. 


\section{L'émergence d'un nouvel acteur : la Coalition pour la société civile} et la démocratie

À la fin des années 1990, la nécessité de créer une nouvelle dynamique s'impose au sein de la nébuleuse internationale (Rist, 2002). Une fois accepté la vision libérale d'un État minimal, il s'avère nécessaire de recréer des structures de planification pour faire face à l'éparpillement des ONG locales pour organiser les politiques qui ont été mises en place. Ce n'est plus forcément au politique que l'on demande de remplir ce rôle, mais aux ONG de s'organiser en «coalition », «réseau » ou «forum». Une opposition au gouvernement se structure aussi sur ce principe. L'histoire de Koalitsia, la Coalition pour la société civile et la démocratie au Kirghizstan, en constitue un excellent exemple. Cette organisation a été créée en 1998 à l'initiative de la fondation américaine du Parti démocrate (NDI) ${ }^{22}$ dirigée par Madeleine Albright. Le NDI a pour objectif la «promotion de la démocratie dans l'intérêt stratégique des USA ${ }^{23}$ », ce qui peut apparaître comme une première ambiguïté. Malgré le discours universaliste américain sur la liberté et la démocratie, la promotion de la démocratie se fait au gré des intérêts stratégiques. C'est par exemple le cas au Turkménistan, où la pression en faveur d'un changement démocratique ne semble pas aller dans le sens des intérêts de Washington. Le National Democratic Institute fait partie d'une organisation publique, le National Endowment for Democracy (NED), chargée de mettre au centre de la politique extérieure étatsunienne la promotion de la démocratie (Guilhot, 2005). C'est un relais du département d'État américain pour assurer concrètement sa volonté d'influence dans le monde. Ce dernier a dépensé plus de 750 millions de dollars au Kirghizstan pour animer ces différents projets de promotion de la démocratie ${ }^{24}$. Washington a consacré, dans le cadre du Freedom Act et de l'Agence nationale de développement (UsAid), 31 millions de dollars uniquement pour l'année 2004 au Kirghizstan. Une partie de cet argent sert à alimenter les activités du NDI, qui est présent dans le pays malgré une représentation très discrète à Bichkek. Son bureau est dirigé par Amy Schultz, qui est une professionnelle de la politique puisqu'elle a été attachée parlementaire de différents députés

22. L'équivalent existe pour le Parti républicain, l'International Republican Institute, ces deux fondations faisant partie du National Endowment for Democracy (NED). Il semblerait qu'il y ait une division du travail par pays ou par thème entre les deux fondations, qui ne se considèrent pas réellement concurrentes sur le terrain.

23. www.ndi.org.

24. $C f$. John Laughland, «The Mythology of People Power: The Glamour of Street Protests Should not Blind us to Reality of Us Back Coups in the Former USSR », The Guardian, $1^{\mathrm{er}}$ avril 2005. 
démocrates et conseillère en communication. Au Kirghizstan, elle s'appuie essentiellement sur des salariés kirghizes qui animent les 18 centres régionaux pour diffuser de l'information et organiser des relations avec des publics ciblés (hommes politiques, jeunes, partis politiques). Le bureau kirghize fait appel à des experts américains (spin doctors) qui interviennent ponctuellement dans la formation et le conseil d'hommes politiques. Ces professionnels de la politique ont notamment joué un rôle clé dans la constitution de la Coalition des partis d'opposition autour Kurmanbek Bakiev et Roza Otumbaeva ${ }^{25}$. Le National Democratic Institute met au centre de sa stratégie l'émergence d'organisations «civiles » locales dites non partisanes, chargées de veiller sur le caractère démocratique de la vie politique. À partir de 1998, NDI s'appuie sur Koalitsia au Kirghizstan et reprend le modèle de coalition d'ONG pour la société civile existant dans la plupart des pays de l'Est $^{26}$. Elle est l'équivalent du CESID ${ }^{27}$ en Serbie, du CVU ${ }^{28}$ en Ukraine, $\mathrm{d}^{\prime}$ ISFED $^{29}$ en Géorgie, qui ont aussi contribué à faire tomber les régimes présidentiels de leurs pays repectifs.

Au moment de sa création, Koalitsia est dirigée par une femme militante, Tolikan Ismaïlova, qui s'oppose très fréquemment à la politique du gouvernement Akaev. Son engagement contribue rapidement à médiatiser son organisation aux niveaux national et international. Après le référendum de 2001 et l'emprisonnement du député de l'opposition Beknazarov, elle décide de se présenter à un scrutin électoral malgré les réticences de la fondation américaine : «Le représentant de NDI m'a convoquée pour me demander de démissionner... je n'ai pas compris pourquoi je devais quitter mon organisation... j'ai répondu que c'était notre organisation et qu'il ne fallait pas qu'il s'immisce dans notre fonctionnement, qu'elle appartenait aux Kirghizes. Le représentant de NDI m'a dit que Koalitsia était une propriété de NDI et m'a obligée à partir... comme nous étions financés à $99 \%$ par eux, j'ai donc laissé la place au jeune Edil Baïsalov, que les Américains avaient choisi pour me remplacer.»

Exercer le pouvoir au sein de cette organisation ne s'appuie pas sur une légitimité issue «d'en bas ». Edil Baïsalov est salarié de son organisation et il n'a pas été élu par les différents membres de la coalition qu'il choisit. Cette organisation n'est donc pas le résultat de forces sociales souhaitant se regrouper dans une

25. Elle a été ambassadrice à Washington.

26. $C f$. tableau en introduction.

27. Centar za Slobodne Izbore i Demokratiju (Centre pour des élections libres et démocratiques).

28. Committee of Voters in Ukraine (Comité des électeurs en Ukraine).

29. International Society for Fair Elections and Democracy (Société internationale pour des élections libres). 
structure paritaire. Très souvent, ces ONG locales ne s'estiment pas l'incarnation d'une force sociale de contestation.

La création des ONG locales résultant souvent d'une orientation définie par les bailleurs de fonds, elle est moins souvent le résultat d'une initiative locale ou d'une préoccupation locale. Le président d'une ONG de Baetov, membre de Koalitsia, explique: «Ici il y a beaucoup de chômage, je possède quelques hectares de terre et un petit troupeau mais cela ne suffit pas pour vivre, alors j'ai fait un projet pour créer une ONG sur l'écologie, il paraît que c'est un thème porteur $[\ldots .$.$] cela peut nous faire un complément de revenu { }^{30}$.»

On pourrait multiplier les exemples de ce type: du côté international l'ONG apparaît être un outil d'influence, mais du côté kirghize elle s'apparente avant tout à une ressource qu'il faut capter. Elle peut également servir des logiques de conquête.

L'activité étrangère est présente dès la formation de Koalitsia mais cela ne signifie pas pour autant qu'il s'agisse d'une organisation aux ordres de Washington car il y a aussi des stratégies locales de manipulation pour capter cette nouvelle ressource. Pour comprendre la recomposition du pouvoir dans ces sociétés, il ne suffit pas de réduire l'ongénisation soit à un phénomène purement exogène soit à une création locale. Il s'agit de la rencontre d'acteurs locaux et transnationaux qui entretiennent des rapports produisant des déplacements de pouvoir. L'éviction de Tolikan Ismaïlova souligne la dépendance financière et politique de Koalitsia car le National Democratic Institute est son bailleur exclusif, mais met aussi en évidence que le financeur ne maîtrise pas les logiques politiques locales. L'actuel président, Edil Baïsalov, reconnaît volontiers l'importance de l'aide extérieure : «nous disposons d'un budget de 110000 euros exclusivement de NDI... je ne fais pas partie de ceux qui dénient le rôle capital des Américains dans les événements de mars, sans le soutien extérieur nous n'existerions pas $^{31}{ }$. Si ce type d'organisation n'a effectivement plus de rapport direct avec le gouvernement kirghize, elle développe néanmoins d'autres formes de dépendance à l'égard d'un nouveau pouvoir : celui d'une fondation politique étrangère. De plus, au-delà du problème de l'autonomie matérielle, se pose aussi la question des procédures pour désigner un représentant légitime. Son leader, Edil Baïsalov, n'a pas été élu par les ONG qu'il entend représenter et est salarié du National Democratic Institute. Koalitsia s'approprie et s'arroge le rôle de représentant d'une société civile contestataire en s'appuyant sur l'existence de son réseau réunissant 170 ONG sur l'ensemble du territoire kirghize, dont la consistance réelle reste à mesurer.

30. Entretien personnel réalisé en octobre 2004.

31. P. Shishkin : «Ripple Effect: In Putin's Backyard, Democrats Stirs », The Wall Street Journal, 25 février 2005. 
Koalitsia occupe néanmoins, par ses actions, une place stratégique dans le paysage politique à partir de l'année 2000. Son action se concentre sur la réalisation de «monitoring électoral », qui consiste à observer les élections, produire des sondages, des statistiques et à organiser la diffusion d'informations électorales auprès des médias nationaux et internationaux. En produisant une information sur le déroulement des élections, elle participe au processus de légitimation/déligitimation d'un pouvoir issu des urnes. Elle met également en place une campagne de participation civique dite positive ${ }^{32}$ consistant à mobiliser les citoyens généralement peu concernés par les élections. Edil Baïsalov organise aussi une campagne dite négative qui consiste à décrédibiliser le gouvernement Akaev en diffusant des informations sur la corruption et l'illégitimité du régime.

Koalitsia est exclusivement composée de citoyens kirghizes. Le parcours de son directeur Edil Baïsalov témoigne de l'émergence de nouvelles élites ayant des références intellectuelles et des expériences humaines différentes de celles qui étaient au pouvoir. Son cursus est intéressant car il est sensiblement le même que celui de milliers de jeunes qui animent aujourd'hui les grandes ONG des pays de l'Est et qui sont investis dans la conquête du pouvoir. Baïsalov organise régulièrement des conférences de presse pour informer la presse internationale de la situation politique du pays. Il jouit d'ailleurs d'une audience particulière auprès du microcosme des organisations internationales, des ambassades et des ONG occidentales. Son organisation joue un rôle déterminant au moment des élections de mars 2005 parce qu'il a la capacité d'observer les élections, de remettre en cause la fiabilité du scrutin électoral et de médiatiser différentes formes locales de contestation.

Des élections sous haute surveillance: ONG transnationales et organisations internationales. - Koalitsia est un élément d'un pouvoir transnational d'un nouveau type qui tend à se généraliser dans certaines parties du monde. Cette ONG s'insère dans une architecture complexe de l'observation électorale au Kirghizstan qui regroupe des acteurs internationaux comme l'OSCE, des acteurs transnationaux comme ENEMO ${ }^{33}$. Au-delà de la diversité des institutions, il y a de nombreux points communs entre eux. L'OSCE a déployé plusieurs centaines d'observateurs en mars 2005 au Kirghizstan. Ce sont des diplomates, des fonctionnaires ou des représentants de la société civile envoyés par les ministères des Affaires étrangères des pays membres de l'organisation ${ }^{34}$. L'OSCE fait donc appel à un important

32. $C f$. compte rendu de Mayeul Kauffman sur l'ouvrage de Gene Sharp.

33. Voir ci-après, European Network of Election Monitoring Organizations, http ://www. enemo.org.ua/

34. Il faut préciser que le Groupe de Shangaï et la CEI ont déployé quelques observateurs au cours des élections de mars 2005, faisant suite aux critiques émises par la Fédération de Russie 
contingent de représentants d'ONG venant d'autres pays de l'Est, comme par exemple des membres du CESID serbe. Radivoje Grujic, responsable du CESID à Loznica et observateur de court terme pour l'OSCE à Bichkek, déclare : «je suis salarié du CESID et je suis devenu expert en élections depuis la révolution de Belgrade. J'ai aussi fait l'Ukraine et $j$ 'attends avec impatience de voir ce que l'on va faire ici. Avant j'ai fait des missions pour ENEMO et maintenant c'est la consécration puisque je suis observateur pour l'OSCE ».

Le microcosme des ambassades occidentales, des organisations internationales et celui des ONG ne sont pas des éléments hermétiques les uns aux autres. Sur le terrain, il existe des relations étroites entre les observateurs regroupés sous différentes bannières. Radivoje Grujic souligne : «nous nous connaissons pour la plupart car nous sommes comme une caravane qui se reforme au gré des élections ».

Au moment de l'observation, malgré la neutralité et l'impartialité affichées de ces différentes organisations, un certain nombre d'observateurs affichent clairement leur intention de participer au déboulonnage d'Akaev. Natalia Toropova, une Ukrainienne salariée du National Democratic Institute (NDI) à Kiev, est observateur de court terme pour l'OSCE et dit: " Je travaille pour le NDI à Kiev, nous avons beaucoup œuvré pour déboulonner Koutchma en Ukraine et je suis ravie de participer à cette mission pour faire la même chose avec Akaev. »

Le scrutin de mars $2005^{35}$ se déroule donc sous une pression maximale pour le gouvernement kirghize, symbolisée par la présence de parfois dix observateurs dans un même bureau de vote. Koalitsia déploie également les siens avant et pendant le scrutin. Pendant la campagne, elle joue un rôle déterminant en médiatisant l'invalidation de candidats, les protestations locales (manifestations, barrages de route, etc.) qui sont immédiatement répercutées par les grands médias internationaux. Koalitsia participe à l'élaboration de la stratégie non violente qui consiste à organiser une campagne civique ayant un volet positif : la formation civique, l'incitation des citoyens à voter, et un volet négatif visant à délégitimer le gouvernement en stigmatisant les pratiques de corruption et de fraudes. De son côté le pouvoir tente de lancer une organisation similaire - Associatsia - pour contrer l'influence de Koalitsia, sans grand résultat. Au moment du scrutin, le réseau d'observateurs de Koalitsia permet de médiatiser des fraudes mais aussi de produire des résultats alternatifs qui viennent contester la validité des résultats offi-

sur le rôle de l'OSCE dans les changements politiques en Ukraine et en Géorgie. La Russie et la Chine s'associent donc pour tenter une riposte à l'architecture transnationale développée par les Occidentaux.

35. Cette élection a été l'occasion de mener une observation participante au sein de l'OSCE comme observateur de court terme dans la région de Naryn. 
ciels. Koalitsia peut compter sur la logistique des 18 centres de NDI pour faire parvenir rapidement les informations par l'internet, le fax ou le téléphone. Après le scrutin, Koalitsia poursuit son travail, organisant des manifestations populaires (notamment la marche de manifestants sur Bichkek en collaboration avec le mouvement de jeunesse Kel-Kel et la coalition d'opposition) et diffusant des informations redoutables contre le gouvernement Akaev.

Koalitsia, Kel-Kel et la coalition d'opposition Ata Jurt articulent et fédèrent les différents collectifs répartis sur l'ensemble du territoire afin de canaliser le mécontentement populaire qui est médiatisé par la radio Azatik (Free Europe) et le journal privé $M S N$ (financé par Freedom House). Ces quatre acteurs contribuent à donner une unicité à un mouvement très éclaté, et participent à l'élaboration et la diffusion des symboles (couleurs, tee-shirts, drapeaux, etc.) incarnant un consensus collectif. À tel point que, au-delà de l'importance de son maillage au niveau local, cette coalition s'apparente à une structure de pouvoir qui part du local avec son tissu d'ONG pour remonter jusqu'au national et qui a la capacité de concurrencer l'État dans sa faculté de produire des résultats électoraux.

Koalitsia s'était préparée à jouer ce rôle depuis plusieurs années. Les membres de l'organisation ont participé à de nombreuses formations et séminaires avec d'autres jeunes issus du mouvement éphémère Kel-kel. La formation, les voyages et les bourses d'études jouent un rôle de premier plan dans l'émergence de ces nouveaux acteurs. Ceux-ci structurent le mécontentement et donnent une cohérence à un mouvement populaire très éclaté.

L'exaspération populaire, qui s'est concentrée au moment des élections législatives de 2005, ne peut pas être réduite à une volonté de réclamer davantage de droits politiques. La mobilisation sociale repose avant tout sur un fort mécontentement provoqué par les conditions d'enrichissement d'une petite élite et le déclassement que subit une bonne partie de la population face à la construction d'un système économico-social de plus en plus inégalitaire. Il semblerait que la paralysie actuelle du nouveau gouvernement montre qu'il n'est pas en mesure de satisfaire ces revendications, amenant de très nombreux Kirghizes à s'interroger sur le sens des événements de mars 2005. Le fétichisme électoral encadré par les nombreux acteurs internationaux donne le sentiment à la population que la démocratisation conduit à une dégradation du niveau de vie et un blocage institutionnel se traduisant par une absence de choix politique pour le futur. La multiplication des consultations électorales et la situation d'inertie provoquent un sentiment de désenchantement parmi la population. 


\section{Fétichisme électoral et stratégie locale de prise de pouvoir}

Dans un premier temps, la prise de pouvoir par Kurmanbek Bakiev est interprétée comme un revers supplémentaire pour la Russie et une victoire américaine en Asie centrale. Le nouvel homme fort a su remarquablement capter l'assistance à la démocratie et le soutien américain. Il a instrumentalisé à merveille ce dispositif complexe pour conquérir le pouvoir. Il décide de maintenir le Parlement issu des élections contestées et organise en priorité des élections présidentielles anticipées (juillet 2005), qui sont un plébiscite dans un contexte électoral où il n'a pas d'adversaire sérieux. Ce scrutin est jugé légitime par Koalitsia et l'OSCE, alors que de nombreuses fraudes se sont déroulées ${ }^{36}$. Kurmanbek Bakiev écarte rapidement Roza Otumbaeva et prend ses distances avec Edil Baïsalov et Koalitsia. Ces derniers rejoignent alors le Parti social-démocrate soutenu par la coopération américaine et entrent dans l'opposition.

Contre toute attente, le nouveau président remet rapidement en question la présence de la base militaire américaine ${ }^{37}$ et fixe parmi ses priorités le renforcement des liens avec la Russie. À l'instar de la Fédération de Russie, il fait voter une loi encadrant les activités des ONG dans son pays. Après plusieurs mois d'une situation ingouvernable avec un Parlement frondeur, il décide d'organiser un référendum sur la Constitution (octobre 2007) pour mettre en place un système électoral de listes qui éviterait la situation d'instabilité politique. Malgré les nombreuses irrégularités relevées par l'OSCE, le référendum valide la nouvelle Constitution et Bakiev appelle immédiatement à l'organisation de nouvelles élections législatives (décembre 2007). La liste proprésidentielle Ak Jol l'emporte largement, le principal parti d'opposition Ata Meken n'obtient aucun siège tandis que le Parti social-démocrate de Roza Otumbaeva et le Parti communiste placent quelques députés. Une nouvelle organisation d'observations électorales émerge, grâce au soutien de NDI, Taza Shailo, qui prend la place de Koalitsia. Taza Shaïlo et l'OSCE dénoncent de nombreuses irrégularités pendant la campagne les amenant à remettre en cause la légitimité du scrutin. D’un autre côté, Moscou et Pékin félicitent Kurmanbek Bakiev pour sa victoire.

Cette fois-ci, le nouvel homme fort du Kirghizstan ne s'appuie plus sur le dispositif de l'assistance à la démocratie, mais se réfère à une autre conception politique, celle véhiculée en Fédération de Russie par Vladimir Poutine, insistant sur

36. $C f$. rapport de l'OSCE.

37. Il a finalement renégocié les conditions financières de la présence de la base militaire américaine tout en rappelant que cette base sert uniquement aux opérations logistiques et humanitaires en Afghanistan. 
la nécessité de rétablir la verticale du pouvoir et assurer la stabilité politique de la région. Il sait qu'il peut également compter sur le Groupe de Shanghai pour reconnaître sa légitimité.

De son côté, le leader de l'opposition et ancien proche d'Askar Akaev, Omurbek Tekebaev, déclare que ce résultat est illégitime et appelle à la création d'un comité révolutionnaire pour faire destituer Bakiev. L'idée des manifestations, des villages de tentes est reprise par la nouvelle opposition. Tekebaev s'est rendu aux États-Unis après les élections pour y glaner un soutien et rencontrer des responsables de la politique extérieure américaine dont Richard Boucher. À son retour, il a déclaré : «j'ai décidé d'ouvrir un bureau représentant notre parti politique à Washington pour permettre aux Kirghizes de l'étranger de jouer un rôle politique au Kirghizstan [...] nous avons décidé aussi de devenir membres de l'Internationale socialiste en pensant qu'une présence en Europe allait nous faciliter une intégration internationale ${ }^{38} \gg$. Cette déclaration montre que la vie politique kirghize repose de plus en plus sur la nécessité de construire des soutiens à l'extérieur.

Côté russe, après une période de désintérêt à l'égard de l'Asie centrale, l'arrivée de Vladimir Poutine en 2001 marque un tournant et un changement de conception. Tout d'abord, ce dernier renforce la théorie du libéral Anatoli Tchoubaïs consistant à penser que la Russie doit maintenir une influence prépondérante dans ces pays en prenant des participations majoritaires dans les entreprises des secteurs économiques stratégiques. Après les attentats du 11 septembre 2001, une période de coopération inédite entre Russes et Américains a lieu en Asie centrale. Vladimir Poutine «autorise » l'installation de la base américaine Manas au Kirghizstan tout en développant une aide militaire renforcée avec ce pays, qui se matérialise par l'ouverture de la base militaire de Kant (2003). Depuis, les liens bilatéraux se renforcent considérablement entre l'ancienne République soviétique et Moscou. Au niveau multilatéral, le nouveau gouvernement russe privilégie les accords non pas au sein d'une CEI moribonde, mais dans le cadre d'une collaboration chinoise au sein de l'Organisation du Groupe de Shanghai.

En conséquence, la situation postrévolutionnaire kirghize tend à démontrer que la thèse simpliste d'un complot américain (Knaziev, 2006) ne tient pas. De plus, l'idée de l'existence d'un groupe local clairement identifié au soutien américain et à la promotion de la démocratie ne résiste pas à l'examen des faits. Les promoteurs de la démocratie tentent d'influencer la réalité politique de ce pays mais les acteurs kirghizes utilisent les ressources de la promotion de la démocratie en fonction aussi de stratégies locales de conquête du pouvoir. Koalitsia a disparu de la

38. Journal Kirghize Reporter, $\mathrm{n}^{\circ}$ 4, 22 février 2008. 
scène publique et son président Edil Baïsalov ${ }^{39}$ s'est lancé en politique au sein du Parti social-démocrate pour tenter de conquérir un siège au Parlement kirghize au cours des élections législatives anticipées de décembre 2007. La nouvelle organisation de monitoring électoral Taza Shaïlo («Élections vraies») a immédiatement émergé par le financement du NDI. De son côté, le leader de la Révolution des tulipes, présenté par les bailleurs de fonds et les médias occidentaux en 2005 comme un "démocrate », a su remarquablement utiliser cette ressource internationale qu'est la promotion de la « démocratie » pour conquérir le pouvoir. Il vient de remporter les élections législatives en utilisant toutes les techniques d'influences (fraudes, pressions administratives) utilisées par son prédécesseur qu'il avait tant décrié. Le contexte politique international a changé et Kurmanbek Bakiev sait qu'il peut compter sur le soutien de Moscou et Pékin pour asseoir son pouvoir.

\section{Conclusion}

Le changement politique intervenu en mars 2005 au Kirghizstan témoigne de profondes mutations initiées au début des années 1990. Ce petit pays s'est lancé dans une captation de ressources tous azimuts le plaçant aussi dans de multiples dépendances et contradictions. Moscou n'est plus comme à l'époque soviétique l'arbitre du combat entre les factions sud et nord du pays. La promotion de la démocratie fait partie des ressources que les acteurs mobilisent dans le jeu politique local. Des relations complexes se sont nouées entre certains acteurs internationaux, transnationaux et des acteurs locaux, qui ont permis l'émergence de nouveaux protagonistes comme Koalitsia. Au moment des élections de mars 2005, Koalitsia n'est que l'un des éléments d'un dispositif plus large regroupant une coalition de partis politiques (Ata Jurt), un mouvement étudiant (Kel-Kel) et des médias privés que l'on qualifie généralement d'acteurs de la « société civile». Ces quatre acteurs locaux branchés sur des réseaux transnationaux ne peuvent ni être réduits à un réveil naturel de la société civile ni à une manipulation extérieure ${ }^{40}$, mais correspondent à de nouvelles influences au sein de la société kirghize. L'existence de cette configuration ne réduit en rien la réalité de la mobilisation populaire mais l'idée selon laquelle le départ du président Akaev serait uniquement dû au mécontentement populaire face à la corruption et au manque de liberté

39. Il a été accusé de graves irrégularités pendant la campagne et a fui avec sa famille au Kazakhstan.

40. Rapport de 1'ICG Kyrgyzstan after the Revolution, 4 mai 2005, cf. www.crisisgroup.org. 
n'apparaît pas suffisante car de nombreux pays souffrant des mêmes symptômes dans le monde auraient été confrontés à un scénario identique. Il ne s'agit pas de sous-estimer ce mécontentement, bien au contraire, mais d'évaluer les acteurs qui ont contribué à canaliser la grogne sociale. C'est finalement un dispositif de pouvoir qui a son efficacité de manière contextuelle pour assurer la circulation du pouvoir sans que l'on puisse dire pour autant qu'elle participe à un processus de démocratisation.

L'analyse de la scène politique kirghize amène à s'interroger sur les relations transnationales qui se tissent entre les multiples acteurs tenant un rôle dans cet espace politique. Le cas kirghize montre que l'ongénisation ne peut se réduire à une émancipation totale de toute forme de pouvoir même si les ONG kirghizes ne dépendent plus de l'État. Koalitsia est à la tête d'une structure réunissant des ONG locales, cette même structure dépend d'organisations transnationales (ENEMO) ou d'ONG internationales ou de fondations étrangères (Soros, NDI) que l'on ne peut simplement réduire à des réseaux neutres dans lesquels n'existent pas de nouvelles formes de dépendance et de hiérarchie. Il y a à cet égard une ambiguïté consubstantielle à l'observation électorale qui se veut normalement neutre alors que les coalitions d'ONG nouent généralement des relations avec un camp. Cette tension se retrouve au sein de l'OSCE, où les Russes refusent de plus en plus de participer à des missions.

La situation kirghize témoigne du développement d'une forme de dispositif de pouvoir transnational qui s'instaure dans de nombreux espaces politiques à travers le monde. Finalement, l'émergence de ces acteurs illustre l'universalisation de l'assistance à la démocratie mais ne garantit en rien l'universalisation de la démocratie.

Cependant, les tensions politiques sur le théâtre kirghize illustrent aussi l'affirmation d'une nouvelle opposition internationale. La référence à la Russie, à la Chine et à l'Organisation de coopération de Shanghai s'affirme comme un nouveau modèle politique qui émerge et entend concurrencer le modèle de la bonne gouvernance et les activités de soft power qui se sont déployés en Asie centrale depuis le début des années 1990. 
HÉRODOTE

SOFT POWER AMÉRICAIN ET MONITORING ÉLECTORAL : LE CAS KIRGHIZE

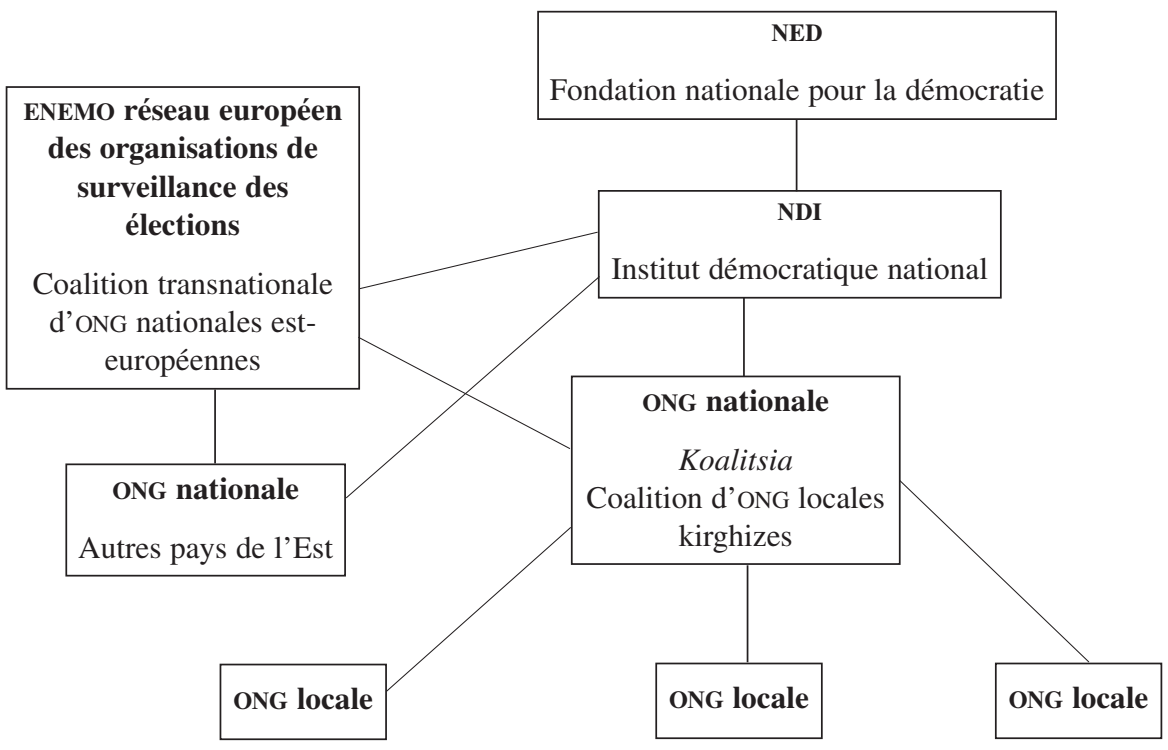

\section{Bibliographie}

Abazov R. (1999) «Policy of economic transition in Kyrgyzstan », Central Asia Survey, vol. $18, \mathrm{n}^{\circ} 2$, p. 197-223.

AKaYeV A. (1995), Kyrghyzstan on the Way to Progress and Democracy, Asto, Bishkek, 1995.

ATLANi L. (2005), «Les ONG à l'heure de la bonne gouvernance », Autrepart, ${ }^{\circ} 35$.

FLORINI A. (2001), Reading Room: The Fird Force: the Rice of Transnational Society, Carnegie Endowment for International Peace, Washington DC.

Guilhot N. (2005), The Democracy Makers: Human Rights and the Politics of Global Order, Columbia University Press, New York.

KNIAZEV A. (2006), Gosudarstvennii perevarot 24 marta 2005 v Kirgizi (Le coup d'État du 24 mars au Kirghizstan), Bichkek.

LARUELle M., Peyrouse S. (2006), Asie centrale, la dérive autoritaire : cinq républiques entre héritage soviétique, dictature et islam, Autrement, Paris.

PETRIC B. (2005), «Post-Soviet Kyrgyzstan or the Birth of Globalised Protectorate », Central Asian Survey, vol. 24, n 3, p. 319-332, septembre. 
POMFRET R., ANDERSON K. (2003), Consequences of Creating Market Economy : Evidence from the Household Survey in Central Asia, Cheltenham, UK.

RIST G. (2002), «Les mots du pouvoir», Les Nouveaux Cahiers de l'IUED, n 13, PUF, Paris, mars.

Ruffin, M. H., Waugh D. C. (dir) (1999), Civil Society in Central Asia, University of Washington Press, Baltimore.

SAMPSON S. (1998), «Exporting Democracy, Preventing Mafia: the Rebirth of Eastern Europe in the Era of Post-Communism », in Karlson K.G., Collective Identities in an Era of Transitions, Londres.

Sharp G. (1992), From Dictatorship to Democracy: A Conceptual Framework for Liberation, Albert Einstein Institute, Boston.

Stiglitz J. (2001), La Grande Désillusion, Fayard, Paris. 